\title{
Testing neutrino decay scenarios with IceCube data
}

\author{
G. Pagliaroli \\ INFN, Laboratori Nazionali del Gran Sasso, Assergi (AQ), Italy \\ A. Palladino \\ Gran Sasso Science Institute, L'Aquila (AQ), Italy \\ F. Vissani \\ INFN, Laboratori Nazionali del Gran Sasso, Assergi (AQ), Italy \\ Gran Sasso Science Institute, L'Aquila (AQ), Italy \\ F.L. Villante \\ L'Aquila University, Physics and Chemistry Department, L'Aquila, Italy \\ INFN, Laboratori Nazionali del Gran Sasso, Assergi (AQ), Italy
}

\begin{abstract}
We test the hypothesis of non-radiative neutrinos decay using the latest IceCube data. Namely, we calculate the track-to-shower ratio expected in IceCube for the normal and inverted neutrino mass hierarchy taking into account the uncertainties in neutrino oscillation parameters. We show that the subset of data with energy above $60 \mathrm{TeV}$ actually excludes the possibility of a neutrinos decay at the 1 sigma level of significance for both neutrino mass hierarchies.
\end{abstract}

Keywords: neutrinos, neutrino decay, neutrino oscillations

\section{Introduction}

The neutrinos decay scenario has been early proposed as an explanation of the solar neutrinos problem[1, 2]. Although the neutrinos decay through radiative processes is well constrained [3], the lifetime limits for non-radiative channels are very weak. Generally researchers focused their attention to non-radiative processes of the kind $v^{\prime} \rightarrow v+X$, where neutrinos decay into possibly detectable neutrinos (or antineutrinos) plus truly invisible particles, $X$, e.g., light scalar or pseudoscalar bosons. As an example, the neutrino decay can take place through the coupling of the neutrino to a very light or massless particle, such as a Majoron, which is also responsible for spontaneous neutrino mass generation [4, 5].

In general, considering a neutrinos source, decay could deplete the flux of neutrinos by the factor,

$$
\exp \left(-\frac{T}{E} \times \frac{m_{i}}{\tau_{i}}\right)
$$

Preprint submitted to Physics Letters $B$ where $m_{i}$ and $\tau_{i}$ are the masses and lifetime of the $i$-th neutrino, subject to decay, $E$ is their energy and $T$ is the time since production. As a consequence, the sensitivity to the unknown ratio $\tau / m$ changes for different distances and energies. Actually the strongest reliable limit for non-radiative processes and for hierarchical masses, namely $\tau / m>1.1 \cdot 10^{-3} \mathrm{~s} / \mathrm{eV}$, is obtained by the nonobservation of solar electron antineutrinos in Kamland [6]. This limit can be in principle improved by several order of magnitude by using the high energy cosmic neutrinos observed in detectors such as IceCube [7].

This intriguing possibility became more interesting on the light of the recent search for High Energy Starting Events (HESE) in IceCube detector that provided the first evidence for high-energy cosmic neutrinos [8, 9, 10]. In three year of data taking [8], 37 events with deposited energies above $30 \mathrm{TeV}$ were observed, relative to an expected background of $8.4 \pm 4.2$ cosmic ray muon events and 6.6 \pm 5.9 atmospheric neutrinos.

Recently, Palladino et al.[11] have discussed the

October 12, 2018 
compatibility of these data with the hypothesis of cosmic origin. In order to reduce the background contamination, authors have considered the subset of events with deposited energy above $60 \mathrm{TeV}$. Using the additional information provided by muon neutrinos passing through the Earth, they show that the observed track-toshower ratio matches expectations for neutrinos of cosmic origin.

In this Letter, we discuss the impact of the nonradiative neutrino decay scenario on the expected IceCube signal in term of the track-to-shower ratio. More precisely, considering the actual uncertainties on oscillation parameters, mass hierarchy and production mechanisms; we use the observed events to constrain the hypothesis of neutrinos decay. As a remarkable result, we show that the subset of data with energy above $60 \mathrm{TeV}$ excludes the possibility of a neutrinos decay at the 1 sigma level of significance for both neutrino mass hierarchies.

\section{Complete Decay Scenario}

To maximize the effect of neutrino decay, we consider the phenomelogy of a generic neutrinos decay and we assume that decays are always complete (i.e., that the exponential factors vanish). This last assumption is reasonable for Cosmic Sources considering that $L \sim 1 \mathrm{Gpc}$ and $E \sim 100 \mathrm{TeV})$, namely

$$
\frac{T / E}{\mathrm{~s} / \mathrm{eV}}=10^{3}
$$

where we used $L=c T$. We call this hypothesis Complete Decay Scenario. In this case, the flux of neutrinos of flavor $\ell$ expected to the Earth is:

$$
\Phi_{\ell}=\sum_{\ell^{\prime}} P_{\ell \ell^{\prime}} \Phi_{\ell^{\prime}}^{0} \quad \text { with } \quad P_{\ell \ell^{\prime}}=\sum_{j=\text { stable }}\left|U_{\ell j}\right|^{2}\left|U_{\ell^{\prime} j}\right|^{2},
$$

where $U$ is the neutrino mixing matrix and the sum only involves stable mass eigenstates. Following [7, 11], we introduce the flavor fractions at Earth (i.e., in the detection point), defined as:

$$
\xi_{\ell} \equiv \Phi_{\ell} / \Phi_{\text {tot }} \quad \text { with } \quad \Phi_{\text {tot }}=\sum_{\ell=a c t i v e} \Phi_{\ell}
$$

In term of this quantity the relation between the flavor ratios at Earth and the ones at source becomes:

$$
\xi_{\ell}=\frac{\sum_{j}\left|U_{\ell j}\right|^{2} \sum_{\ell^{\prime}}\left|U_{\ell^{\prime} j}\right|^{2} \xi_{\ell^{\prime}}^{0}}{\sum_{j, \ell}\left|U_{\ell j}\right|^{2} \sum_{\ell^{\prime}}\left|U_{\ell^{\prime} j}\right|^{2} \xi_{\ell^{\prime}}^{0}}
$$

where the indeces $j$ and $\ell^{\prime}$ run over stable mass eigenstates and active flavors, respectively. The expression in Eq.(3) also holds in the case where sterile neutrino states are included. Different production mechanisms generate a different initial flavor content at the source $\left(\xi_{e}^{0}: \xi_{\mu}^{0}: \xi_{\tau}^{0}\right)$. For example the most studied production processes are:

$(1 / 3: 2 / 3: 0)$ for $\pi$ decay;

$(1 / 2: 1 / 2: 0)$ for charmed mesons decay;

(1:0:0) for $\beta$ decay of neutrons;

$(0: 1: 0)$ for $\pi$ decay with damped muons.

For cosmic neutrinos, without neutrino decay processes, the final flavor ratios are expected to be very near to $\left(\xi_{e}: \xi_{\mu}: \xi_{\tau}\right) \sim(1 / 3: 1 / 3: 1 / 3)$, independently on the specific production mechanism [12, 13, 14, 15, 16]. On the other hand, turning on the "invisible" decay process discussed above, the flavor ratios at the Earth change dramatically [7]. In the case of only one stable mass eigenstate $j$ and for the standard scenario with three actives neutrinos, the expression of Eq.(3) becomes

$$
\xi_{\ell}=\left|U_{\ell j}\right|^{2},
$$

where the sensitivity to the different production mechanisms has disappeared. The simplified expression in Eq. (4) is due to the fact that only one mass eigenstate is stable and all the others have had time to decay, so that the universe is populated only by neutrinos in the state of mass $j$ and obviously the content of flavor $\ell$ expected to the detector is $\left|U_{\ell j}\right|^{2}$.

Assuming normal mass hierarchy, the simplest case is to consider that both $v_{3}$ and $v_{2}$ decay, so that only the lightest eigenstate $v_{1}$ survives. In this case the final flavor ratios are expected to be $\left(\left|U_{e 1}\right|^{2}:\left|U_{\mu 1}\right|^{2}\right.$ : $\left.\left|U_{\tau 1}\right|^{2}\right) \sim(0.68: 0.21: 0.11)$. For inverted mass hierarchy, a similar expression holds but the stable state in this case is $v_{3}$, i.e. the final flavor ratios are expected to be $\left(\left|U_{e 3}\right|^{2}:\left|U_{\mu 3}\right|^{2}:\left|U_{\tau 3}\right|^{2}\right) \sim(0.02: 0.57: 0.41)$. In order to evaluate these ratios, we used the best-fit values for the oscillation parameters reported in [17].

\section{Track-to-Shower Ratio}

To compare the expectations with the data collected by IceCube we need to convert the flavor neutrino ratio expected to the Earth in the corresponding observable quantity, i.e. the track-to-shower ratio including the detector response. Indeed, by exploiting the event topology, the flavor discrimination is possible, in principle, in IceCube detector. The HESE data collected by IceCube during $T=988$ days, encompass two different topologies: 'shower' topology, that includes neutral current (NC) interactions of all neutrino flavors and charged current (CC) interactions of $v_{\mathrm{e}}$ and $v_{\tau}$; 'track' 


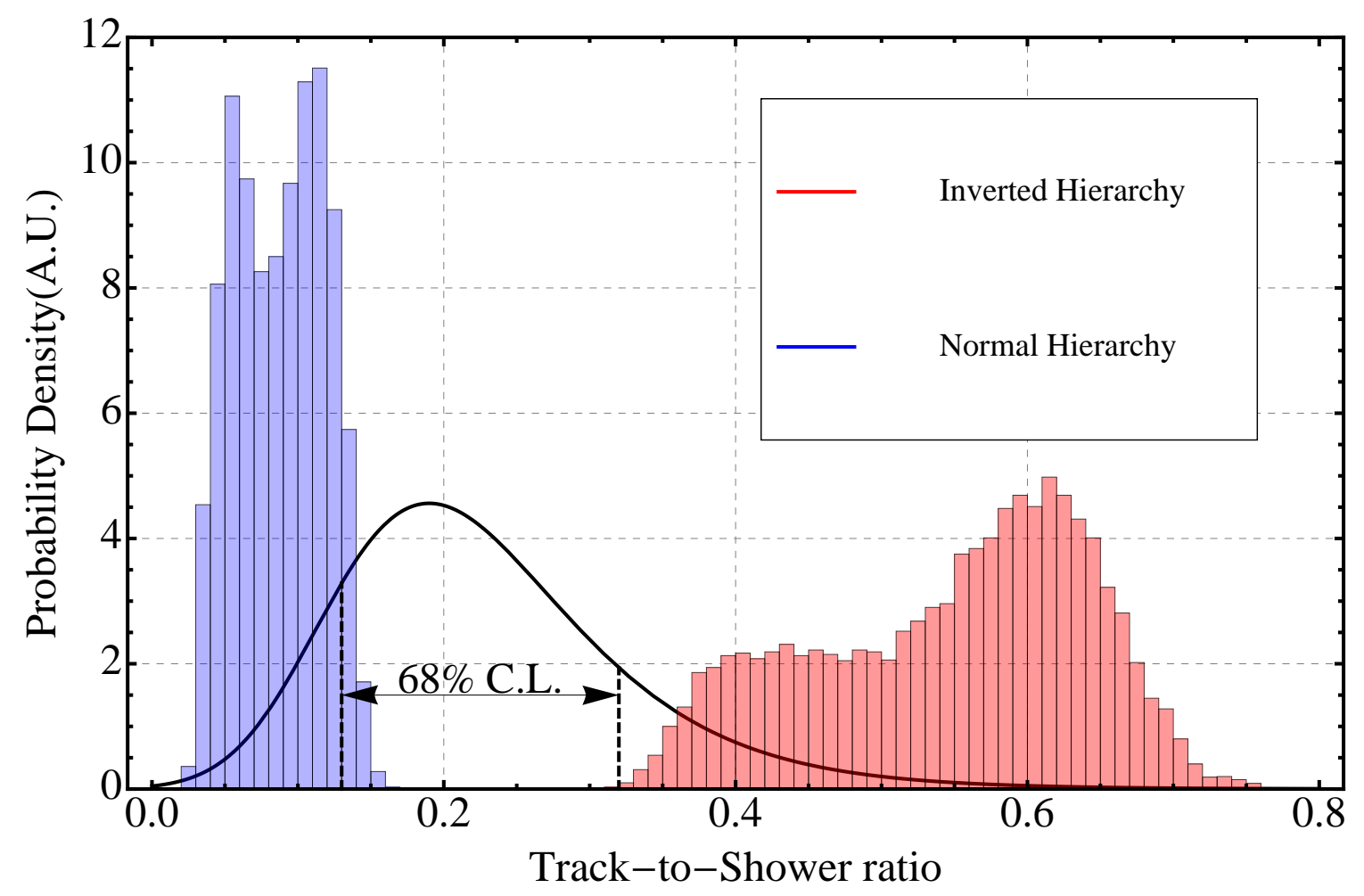

Figure 1: Expected track-to-shower ratio for decaying cosmic neutrinos. The distributions show the effect of uncertainties in the neutrino oscillation parameters. The left (resp. right) distribution is obtained for normal (resp. inverse) hierarchy. The black line shows the normalized likelihood function of the IceCube data-set discussed in the text.

topology produced by $\mathrm{CC}$ interactions of $v_{\mu}$. Thus the track-to-shower ratio is the observable quantity that is most directly related to the flavor ratio at the Earth as discussed in several recent papers [11, 18, 19, 20, 21].

In particular Palladino et al. [11] described the way to calculate the number of showers $N_{\mathrm{S}}$ and tracks $N_{\mathrm{T}}$ in the IceCube detector for an isotropic flux $\Phi_{\ell}$ of neutrinos and antineutrinos described as

$$
\Phi_{\ell}(E)=\frac{F_{\ell} \cdot 10^{-8}}{\mathrm{~cm}^{2} \mathrm{~s} \mathrm{sr} \mathrm{GeV}} \cdot\left(\frac{\mathrm{GeV}}{E}\right)^{\alpha}
$$

where the factors $F_{\ell}$ are (non-negative) adimensional coefficients and $\alpha$ is the spectral index. The number of showers and tracks can be obtained by using the following equations:

$$
\begin{aligned}
& N_{\mathrm{S}}=T\left\{F_{e} c_{e}+F_{\tau} c_{\tau}+F_{\mu} c_{\mu}\left(1-p_{\mathrm{T}}\right)\right\}, \\
& N_{\mathrm{T}}=T F_{\mu} c_{\mu} p_{\mathrm{T}},
\end{aligned}
$$

where $T$ is the time of exposure and the coefficients $c_{l}=$ $4 \pi \int d E E^{-\alpha} A_{\ell}(E)$ include the detector effective areas $A_{\ell}(E)[10]$ and the energy dependence of the fluxes. The extra factor $p_{\mathrm{T}}$ in Eq. 7 accounts for the fraction of the effective area $A_{\mu}(E)$ giving tracks, this fraction is about $\sim 0.8$ and mildly dependent on energy as discussed in [11]. So that, the following useful expression to obtain the track-to-shower ratio from the final neutrino flavor ratios can be obtained generally:

$$
\frac{N_{\mathrm{T}}}{N_{\mathrm{S}}}=\frac{\xi_{\mu}}{c_{1}+c_{2} \xi_{\mu}+c_{3} \xi_{\tau}},
$$

in which $c_{j}$ are given, with good approximation, by:

$$
\begin{gathered}
c_{1}=\frac{c_{e}}{p_{\mathrm{T}} \cdot c_{\mu}} \simeq 2.23+1.15(\alpha-2)+0.46(\alpha-2)^{2} \\
c_{2}=\frac{\left(1-p_{\mathrm{T}} c_{\mu}-c_{e}\right.}{p_{\mathrm{T}} \cdot c_{\mu}} \simeq-1.99-1.15(\alpha-2)-0.46(\alpha-2)^{2} \\
c_{3}=\frac{c_{\tau}-c_{e}}{p_{\mathrm{T}} \cdot c_{\mu}} \simeq-0.56-0.78(\alpha-2)-0.40(\alpha-2)^{2}
\end{gathered}
$$

In the context of neutrino decay scenario described above and fixing the spectral index $\alpha=2$, we have that for Normal mass hierarchy Eq. (8) becomes:

$$
\frac{N_{\mathrm{T}}}{N_{\mathrm{S}}}=\frac{\left|U_{\mu j}\right|^{2}}{2.3-2.0\left|U_{\mu j}\right|^{2}-0.6\left|U_{\tau j}\right|^{2}},
$$

where the index $j$ indicates the stable mass eigenstate. For Normal Mass Hierarchy $j=1$ and substituting the 
best-fit values of the oscillation parameters we obtain $\left(\frac{N_{\mathrm{T}}}{N_{\mathrm{S}}}\right)^{N H}=0.12$. For Inverted Mass Hierarchy $j=3$ and this fraction becomes $\left(\frac{N_{\mathrm{T}}}{N_{\mathrm{S}}}\right)^{I H}=0.62$. The impact of the spectral index of neutrino spectrum is not so dramatic, in fact if we consider a greater slope, like $\alpha=2.6$, these number become $\left(\frac{N_{\mathrm{T}}}{N_{\mathrm{S}}}\right)^{N H}=0.09$ and $\left(\frac{N_{\mathrm{T}}}{N_{\mathrm{S}}}\right)^{I H}=0.56$. Instead, a much more important contribution is given by uncertainties on neutrino oscillations parameters, that allow these numbers to fluctuate in a more large interval. To correctly account for these uncertainties we construct likelihood distributions of $\sin ^{2} \theta_{12}, \sin ^{2} \theta_{13}, \sin ^{2} \theta_{23}$ and $\delta$ from the $\Delta \chi^{2}$ profiles given by [17]. Namely, we assume that the probability distributions of each parameter are provided by $\mathcal{L}=\exp \left(-\Delta \chi^{2} / 2\right)$. Then, we combine the various likelihood functions assuming negligible correlations and we determine the probability distributions of $N_{\mathrm{T}} / N_{\mathrm{S}}$ by MonteCarlo extraction of the oscillation parameters. The resulting Probability Density (PD) distributions are reported in Fig.(1) with a color code blue (resp. red) for Normal (resp. Inverted) Mass Hierachy.

The predicted PDs can be compared with the distribution of the track-to-shower ratio preferred by the IceCube data. In the first column of Tab. (1) we report the events observed by IceCube in the HESE data set [8] with a deposited energy above $60 \mathrm{TeV}$. We devided the events by topology and we includ, in brackets, the respective number of estimated background events due to atmospheric neutrinos and muons.

The number of tracks $N_{\mathrm{T}}$ and showers $N_{\mathrm{S}}$ which have to be ascribed to extraterrestrial sources can be estimated from the Poisson likelihood functions:

$$
\mathcal{L}\left(N_{\mathrm{i}}\right) \propto \lambda_{\mathrm{i}}^{n_{\mathrm{i}}} \times e^{-\lambda_{\mathrm{i}}}
$$

where $\lambda_{\mathrm{i}}=N_{\mathrm{i}}+b_{\mathrm{i}}$ and the index $\mathrm{i}=\mathrm{T}, \mathrm{S}$ is used to refer to track and shower events and $n_{\mathrm{i}}$ are the observed events. We are assuming that the prompt atmospheric neutrinos give negligible contributions, as it required by the spectral and arrival angles distributions of IceCube events. A completely equivalent and independent information can be obtained by the recently released IceCube data on passing muons [22], as already done by Palladino et al. in [11]. In the assumption of $E^{-2}$ neutrino

Table 1: The total number of events, $n_{\mathrm{i}}$, with the expected background, $b_{i}$, seen at IceCube, in the energy range $60 \mathrm{TeV} \leq E_{d e p} \leq 3 \mathrm{PeV}$.

\begin{tabular}{|c|c|c|}
\hline & $n_{\mathrm{i}}\left(b_{i}\right) 988$ days & $n_{\mathrm{i}}\left(b_{i}\right) 1460$ days \\
\hline Track & $4(2.1)$ & $8(3.1)$ \\
\hline Shower & $16(0.7)$ & $25(1.0)$ \\
\hline Total & $20(2.8)$ & $33(4.1)$ \\
\hline
\end{tabular}

spectrum for the passing muons, the flux normalization corresponds to $F_{\mu}=1.01 \pm 0.35$ and taking into account the equivalence between $F_{\mu}$ and $N_{T}$ expressed by the following equation (in which $T$ is the exposure time):

$$
\begin{aligned}
& N_{\mathrm{S}}=\left(2.94 \times F_{e}+0.33 \times F_{\mu}+2.20 \times F_{\tau}\right) \cdot T \\
& N_{\mathrm{T}}=1.31 \times F_{\mu} \cdot T
\end{aligned}
$$

we can include also this information in our analysis by constructing a combined likelihood, given by the product of the 2 Poisson likelihoods for $N_{\mathrm{T}}$ and $N_{\mathrm{S}}$ and of the Gaussian likelihood for $F_{\mu}$ (i.e. $N_{T}$ ). Then we extract the bounds on the track-to.shower ratios of events ascribed to cosmic neutrinos by marginalizing with respect to the total number of events. We obtain:

$$
\frac{N_{\mathrm{T}}}{N_{\mathrm{S}}}=0.18_{-0.05}^{+0.13} \quad[988 \text { days }]
$$

where the error was obtained by integrating out symmetrically $(1-\mathrm{CL}) / 2$ on both sides of the $N_{\mathrm{T}} / N_{\mathrm{S}}$ distribution using a confidence level $\mathrm{CL}=68.3 \%$.

The normalized likelihood distribution of the HESE data is reported in Fig.(1) with a black line and the 1 sigma region is also indicated to easily see its sovrapposition with the predicted PDs in the neutrino decay scenario.

\section{Discussion and conclusion}

Repeating the analysis with 4 years data, under the hypothesis that the background is simply proportional to the exposure time, we found a new range given by:

$$
\frac{N_{\mathrm{T}}}{N_{\mathrm{S}}}=0.20_{-0.05}^{+0.10} \quad[4 \text { years }]
$$

We can notice that this interval is compatible with that found for the 3 year[11] also if the uncertainties are slightly smaller with new data; the track-to-shower of 4 years is more favorable for pions decay at the source, respect to that obtained with 3 years data. In conclusion, it is important to remark that the non-radiative neutrino decays are excluded at least at 1 sigma for both neutrino hierarchies and for both sets of observed data. Moreover these are general considerations and have a poor dependence from the slope of the spectrum of neutrinos.

\section{References}

[1] J. N. Bahcall, N. Cabibbo and A. Yahil, Phys. Rev. Lett. 28 (1972) 316.

[2] S. Pakvasa and K. Tennakone, Phys. Rev. Lett. 28 (1972) 1415.

[3] K. A. Olive et al. [Particle Data Group Collaboration], Chin. Phys. C 38 (2014) 090001. 
[4] Y. Chikashige, R. N. Mohapatra and R. D. Peccei, Phys. Lett. B 98 (1981) 265

[5] G. B. Gelmini and M. Roncadelli, Phys. Lett. B 99 (1981) 411.

[6] K. Eguchi et al. [KamLAND Collaboration], Phys. Rev. Lett. 92 (2004) 071301 |hep-ex/0310047|.

[7] J. F. Beacom, N. F. Bell, D. Hooper, S. Pakvasa and T. J. Weiler, Phys. Rev. Lett. 90 (2003) 181301 [hep-ph/0211305].

[8] M. G. Aartsen et al. [IceCube Collaboration], Phys. Rev. Lett. 113, $101101(2014)$

[9] M. G. Aartsen et al. [IceCube Collaboration], Phys. Rev. D 91, 022001 (2015)

[10] M. G. Aartsen et al. [IceCube Collaboration], Science 342, 1242856 (2013)

[11] A. Palladino, G. Pagliaroli, F. L. Villante and F. Vissani, Phys. Rev. Lett. 114 (2015) 17, 171101

[12] J. G. Learned and S. Pakvasa, Astropart. Phys. 3 (1995) 267 hep-ph/9405296. hep-ph/9408296].

[13] H. Athar, M. Jezabek and O. Yasuda, Phys. Rev. D 62 (2000) 103007 [hep-ph/0005104].

[14] J. F. Beacom, et al. Phys. Rev. D 68 (2003) 093005 [E-ibid. D 72 (2005) 019901]; M. L. Costantini and F. Vissani, Astropart. Phys. 23 (2005) 477; F. L. Villante and F. Vissani, Phys. Rev. D 78 (2008) 103007.

[15] F. Vissani, G. Pagliaroli and F. L. Villante, JCAP 1309, 017 (2013) |arXiv:1306.0211 [astro-ph.HE]].

[16] L. Fu, C. M. Ho and T. J. Weiler, arXiv:1411.1174 [hep-ph].

[17] M. C. Gonzalez-Garcia, M. Maltoni and T. Schwetz, JHEP 1411, 052 (2014)

[18] O. Mena, S. Palomares-Ruiz and A. C. Vincent, Phys. Rev. Lett. 113, no. 9, 091103 (2014)

[19] S. Palomares-Ruiz, O. Mena and A. C. Vincent, arXiv:1411.2998 [astro-ph.HE]

[20] M. G. Aartsen et al. [IceCube Collaboration], arXiv:1502.03376 [astro-ph.HE].

[21] S. Palomares-Ruiz, A. C. Vincent and O. Mena, arXiv:1502.02649 [astro-ph.HE].

[22] C. Weaver, Spring APS Meeting, Savannah, Georgia (2014). M. G. Aartsen et al. [IceCube Collaboration], 